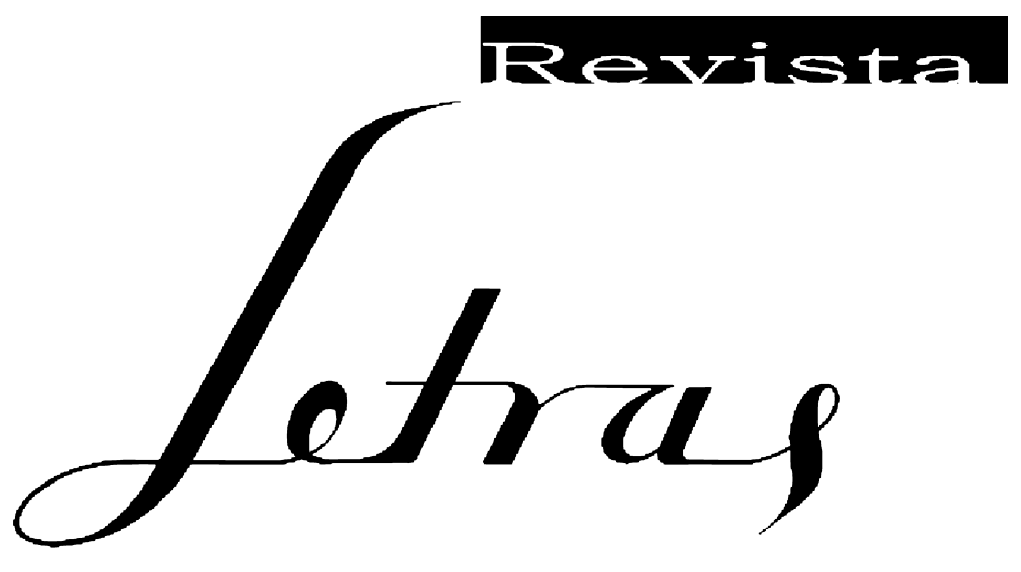




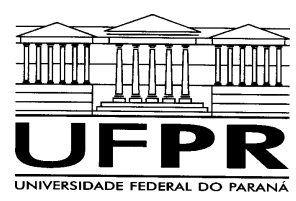

Reitor

Zaki Akel Sobrinho

Vice-Reitor

Rogério Mulinari

Diretor da Editora UFPR

Gilberto de Castro

\section{Revista Letras}

Publicação quadrimestral do Curso de Letras da UFPR

http://calvados.c3sl.ufpr.br/ojs2/index.php/letras

A Revista Letras está indexada nos seguintes índices bibliográficos: 1. Internationale Bibliographie der Rezensionen Wissenschftlicher Literatur/International Bibliography of Book Reviews of Scholarly Literature; 2. Linguistics and Language Behavior Abstracts; 3. MLA-International Bibliography of Books and Articles on Modern Languages and Literatures; 4. Social Planning, Policy and Development Abstracts; 5. Sociological Abstracts; 6. Ulrich's International Periodicals Directory; 7. CLASE - Citas Latinoamericanas en Ciencias Sociales y Humanidades.

Editor: Rodrigo Tadeu Gonçalves

Secretária editorial: Mariana Bordignon Strachulski de Souza

Coordenação da área de Literatura: Renata Telles

Coordenação da área de Linguística: Lígia Negri

\section{Conselho Editorial}

Antonio Dimas (USP), Beatriz Gabbiani (Universidad de la República do Uruguay), Carlos Alberto Faraco (UFPR), Carlos Costa Assunção (Universidade de Trás-os-Montes e Alto Douro), Elena Godoi (UFPR),

Filomena Yoshie Hirata (USP), Gilda Santos (UFRJ), José Borges Neto (UFPR),

Júlio Cesar Valladão Diniz (PUC-RJ), Lígia Negri (UFPR), Lúcia Sá (Manchester University), Lucia Sgobaro Zanette (UFPR), Maria Lucia de Barros Camargo (UFSC),

Marília dos Santos Lima (UNISINOS), Mauri Furlan (UFSC), Maurício Mendonça (UFPR),

Raquel Salek Fiad (Unicamp), Rodolfo A. Franconi (Darthmouth College), Rodolfo Ilari (Unicamp)

\section{Consultores ad hoc}

Célia Maria Arns de Miranda (UFPR), Guilherme Gontijo Flores (UFPR), Klaus Eggensperger (UFPR), Liana Leão (UFPR), Mauricio Cardozo (UFPR), Patrícia da Silva Cardoso (UFPR), Walter Lima Torres (UFPR).

\section{Conselho Consultivo}

Adalberto Müller (UFF), Álvaro Faleiros (USP), Brunno Vinicius Gonçalves Vieira (Unesp-Araraquara), Fernando Cabral Martins (Universidade Nova de Lisboa), Helena Martins (PUC-Rio), Irene Aron (USP), Isabella Tardin Cardoso (Unicamp), Juliana Perez (USP), Luciana Villas Boas (UFRJ),

Márcia Martins (PUC-Rio), Maria Irma Hadler Coudry (Unicamp), Mattew Leigh (University of Oxford), Patrick Farrell (University of California/Davis).

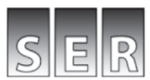

Sistema Eletrônico de Revistas - SER

Programa de Apoio à Publicação de Periódicos da UFPR

Pró-Reitoria de Pesquisa e Pós-Graduação

www.prppg.ufpr.br

O Sistema Eletrônico de Revistas (SER) é um software livre e permite a submissão de artigos e acesso às revistas de qualquer parte do mundo. Pode ser acessado por autores, consultores, editores, usuários, interessados em acessar e obter cópias de artigos publicados nas revistas. O sistema avisa automaticamente, por e-mail, do lançamento de um novo número da revista aos cadastrados. 


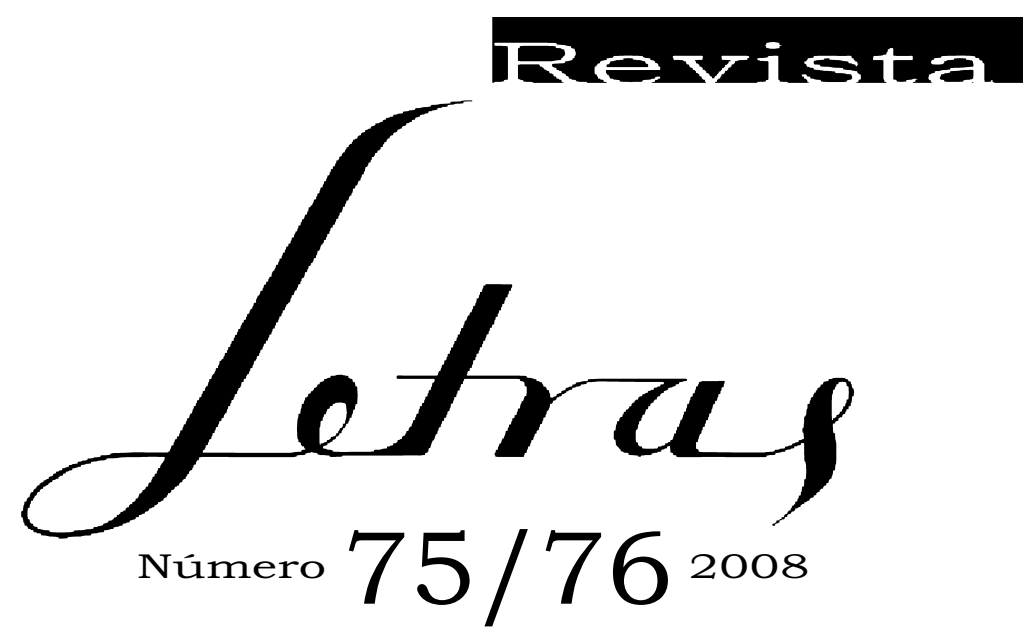

Edityar 


\author{
EDITORA UFPR \\ R. João Negrão, 280, $2^{\circ}$ andar \\ tel./fax (41) 3360-7489 / 3360-7486 \\ Caixa Postal 17.309 - 80.010-200 - Curitiba - Paraná - Brasil \\ www.editora.ufpr.br \\ editora@ufpr.br \\ Coordenação editorial: Daniele Soares Carneiro \\ Revisão dos textos em \\ Português: Vanessa Andrade \\ Inglês: Vera Lúcia Roloff \\ Espanhol: Terumi K. Villalba \\ Francês: Nathalie Anne-Marie Dessartre Mendonça \\ Projeto Gráfico e Capa: Rachel Cristina Pavim \\ Editoração eletrônica: Editora Progressiva Ltda.
}

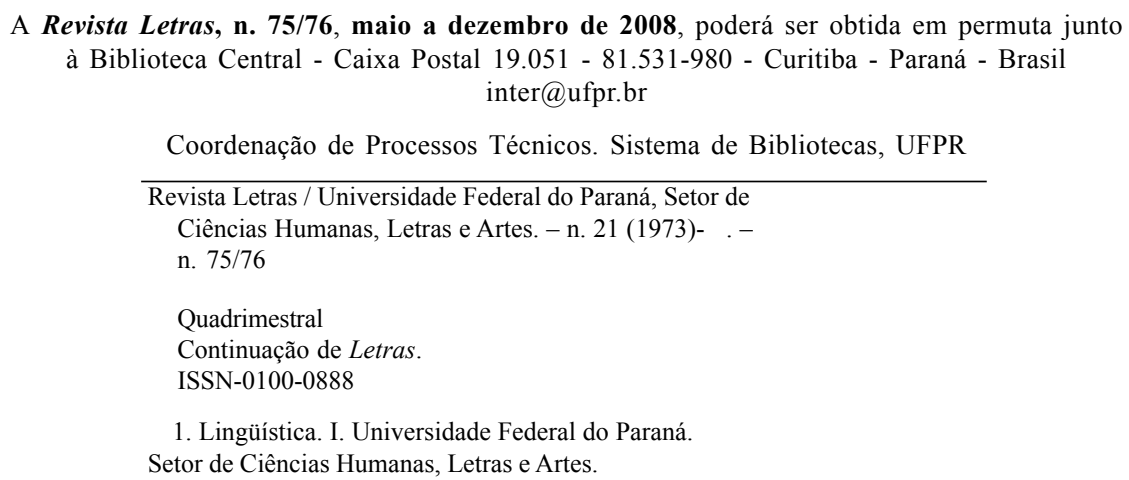

\title{
FUNDACÃO ARAUCARII
}

PRINTED IN BRAZIL

Curitiba, 2009

PEDE-SE PERMUTA

WE ASK FOR EXCHANGE 


\section{ApResentaÇÃo \\ Presentation}

Após alguns problemas que causaram atrasos na publicação da Revista Letras, encontramo-nos em um momento positivo de trabalho e esforço para a regularização da série. Para isso, decidimos publicar este volume duplo. Tendo reformulado nosso conselho editorial, apresentamos os números de maio a dezembro de 2008 em um único volume, que conta com as costumeiras seções de estudos literários e estudos linguísticos.

Na seção de estudos literários, selecionamos seis artigos. Temos o artigo de Marcio Roberto Soares Dias sobre Carlos Drummond de Andrade e sua relação com Belo Horizonte. Em seguida, Francisco Innocêncio nos apresenta um texto sobre a ficção de Valêncio Xavier. O artigo de Rodrigo Guimarães Silva apresenta uma análise do princípio de ordenação nos textos do Marquês de Sade e de Arthur Bispo do Rosário, e Soraya Ferreira Alves escreve sobre Virginia Woolf. Em seguida, temos o artigo de Sigrid Renaux sobre Paul Auster através de uma análise bakhtiniana. A seção se encerra com o artigo de Mail Marques de Azevedo sobre Jamaica Kincaid. Os artigos publicados aqui mostram uma variedade de abordagens teórico-críticas e de autores analisados que caracteriza a orientação editorial de nosso periódico.

A seção de estudos linguísticos selecionou sete artigos para publicação. Iniciamos com o artigo de Marco Antonio Martins, que propõe uma análise diferenciada sobre a questão do tempo na linguística contemporânea, de Saussure ao modelo gerativo minimalista. Xosé Soto Andion apresenta um artigo em espanhol que analisa o verbo "quedar" em galego com foco em esquemas sintático-semânticos. O artigo seguinte, de Marina Rosa Ana Augusto, consiste de comentários sobre outro artigo, de Ana Carolina N. de Aguiar \& Cilene Rodrigues, sobre quantificação em sintagmas determinantes e topicalização. Maria Cristina Figueiredo Silva e Izabel Christine Seara figuram, na sequência, com um artigo sobre entoação em sentenças com estrutura do tipo SV neutras em português brasileiro. Em seguida, temos um artigo de Iara Bemquerer Costa sobre linguística textual focalizado na relação entre gêneros textuais e suporte. $O$ artigo seguinte oferece ao leitor reflexões semânticas sobre sintagmas cardinais complexos e implicaturas escalares. Por fim, Maria Marta Furlanetto nos oferece um artigo sobre redundâncias na escrita formal em português brasileiro a fim de verificar inclusive consequências para o ensino de língua materna. Mais uma vez, a seção apresenta grande variedade de orientações teóricas sem perder de vista a nossa política editorial de qualidade da publicação.

Rodrigo Tadeu Gonçalves

$O$ editor 



\section{Número 75/76, maio/dez. 2008 - Editora UFPR}

Curitiba - Paraná - Brasil

\section{SUMÁRIO / SUMMARY}

\section{Estudos Literários/ Literary Studies}

11 As estrelas do céu de Belo Horizonte são incompreensíveis The stars in the sky of Belo Horizonte are incomprehensible Marcio Roberto Dias

37 A prostituta japonesa e o homem de Baçorá: o fracasso do leitor como estratégia textual na ficção de Valêncio Xavier The Japanese prostitute and the man from Basra: the failure of the reader as a textual strategy in Valêncio Xavier's fiction

Francisco R. S. Innocêncio

51 Para além do princípio de ordenação: Marquês de Sade e Arthur Bispo do Rosário

Beyond the organization's principle: Marques de Sade and Arthur Bispo do Rosário

Rodrigo Guimarães Silva

67 Between the acts: o ato final d(iag)ramático-escritural de Virginia Woolf Between the acts: Virginia Woolf's d(iag)ramatic-scriptural final act Soraya Ferreira Alves

79 Oracle night: a bakhtinian reading of Paul Auster's metafictional narrative

Oracle Night: uma leitura bakhtiniana da narrativa metaficcional de Paul Auster

Sigrid P.M.L.S. Renaux

93 A expansão da representatividade do "eu" no discurso autobiográfico de Jamaica Kincaid The expansion of self-representation in Jamaica Kincaid's autobiographical discourse Mail Marques Azevedo 


\section{Estudos Linguísticos / Linguistic Studies}

113 o Tempo da linguística

The time of linguistics

Marco Antonio Martins

127 Entre la estatividad y el dinamismo: los esquemas con quedar en gallego Between statics and dynamism: Structures with quedar in the Galicien language

Xosé Soto Andión

157 Comentários a respeito do artigo: "Quando expressões nominais quantificadas podem ser topicalizadas" de Ana Carolina N. de Aguiar \& Cilene Rodrigues

Some comments on Aguiar \& Rodrigues's article 'Quando Expressóes Nominais Quantificadas podem ser topicalizadas'

Marina Rosa Ana Augusto

171 Mais sobre a entoação de sentenças com ordem SV

More on the intonation of SV sentences

Maria Cristina Figueiredo Silva

Izabel Christine Seara

183 Contribuições ao debate sobre a relação entre gêneros textuais e suporte Contributions to the debate about the relation between genres and medium

Iara Bemquerer Costa

197 Sintagmas Cardinais Complexos e Implicaturas Escalares Complex cardinal noun phrases and scalar implicatures Marcelo Ferreira

213 As "redundâncias" são mesmo redundâncias? Uma perspectiva discursiva "Redundancies" are really redundancies? A discursive perspective Maria Marta Furlanetto 